\title{
Utilizing the Discrete Element Method for the Modeling of Viscosity in Concentrated Suspensions - Supporting Information
}

\author{
Martin Kroupa, Michal Vonka, Miroslav Soos, and Juraj Kosek* \\ University of Chemistry and Technology Prague, Department of Chemical Engineering, \\ Technicka 5, 16628 Prague 6, Czech Republic \\ E-mail: Juraj.Kosek@vscht.cz
}

\section{Interactions between the particles}

\section{Tangential interactions}

In this section we describe all forces and torques, caused by the interaction of the particle with other particles, which act in the direction tangential to the surface of the particle. We provide the formulas for the description of the resistance to sliding, rolling and twisting of particles. The implementation of the respective resistance models into DEM was carried out by Marshall $(2009)^{1}$ and we adopted it for the purposes of this work. Other formulations of tangential forces can be found in the literature. ${ }^{2,3}$ These usually provide only the resistance to sliding in a manner similar to that proposed by Marshall (2009). Therefore, we decided to use the description of Marshall (2009), as being more complete in terms of the possible modes of tangential interactions.

The sliding is caused by moving of the surfaces of the touching particles in the opposite direction and both rotation and translation can contribute to it. Let us first define $\mathbf{v}_{i}^{\mathrm{c}}$ as the 
surface velocity of particle $i$ at the contact point with particle $j$ :

$$
\mathbf{v}_{i}^{\mathrm{c}}=\mathbf{v}_{i}+\Omega_{i} \times \mathbf{r}_{i}
$$

where $\times$ denotes the cross product of the vectors and $\mathbf{r}_{i}=R_{\mathrm{p}}^{i} \mathbf{n}$ is the vector pointing from the center of the particle $i$ to the contact point (assuming that $\mathbf{n}$ is the unit normal vector pointing from the center of particle $i$ to the center of particle $j$ ). For particle $j$ it is: $\mathbf{r}_{j}=-R_{\mathrm{p}}^{j} \mathbf{n}$. Then the relative velocity $\left(\mathbf{v}_{i j}^{\mathrm{r}}\right)$ of the surfaces of particles $i$ and $j$ at the contact point is defined as follows:

$$
\mathbf{v}_{i j}^{\mathrm{r}}=\mathbf{v}_{i}^{\mathrm{c}}-\mathbf{v}_{j}^{\mathrm{c}} .
$$

We can proceed to the definition of the so-called slip velocity $\left(\mathbf{v}_{i j}^{\mathrm{s}}\right)$ :

$$
\mathbf{v}_{i j}^{\mathrm{s}}=\mathbf{v}_{i j}^{\mathrm{r}}-\left(\mathbf{v}_{i j}^{\mathrm{r}} \cdot \mathbf{n}\right) \mathbf{n},
$$

where $\cdot$ denotes the dot product of the vectors. Here $\mathbf{v}_{i j}^{\mathrm{s}}$ is the projection of $\mathbf{v}_{i j}^{\mathrm{r}}$ tangent to the particle surface at the contact point. Unit vector in the direction of $\mathbf{v}_{i j}^{\mathbf{s}}$ is given by: $\mathbf{t}_{i j}^{\mathrm{s}}=\frac{\mathbf{v}_{i j}^{\mathrm{s}}}{\left|\mathbf{v}_{i j}^{\mathrm{s}}\right|}$.

According to Marshall (2009), the sliding resistance of the particles can be described by the spring-slider model. ${ }^{1}$ Unless the sliding force $\left(F_{i j}^{\mathrm{s}}\right)$ between particles $i$ and $j$ reaches the critical value $\left(F_{i j}^{\mathrm{s}, \text { crit }}\right)$, the sliding is absorbed by the spring. When $\left|F_{i j}^{\mathrm{s}}\right| \geq F_{i j}^{\mathrm{s}, \text { crit }}$ then the sliding force is limited to the critical value. The critical force $F_{i j}^{\text {s,crit }}$ is obtained from the normal force $\left(F_{i j}^{\mathrm{n}}\right)$ between particles $i$ and $j$ as follows:

$$
F_{i j}^{\mathrm{s}, \mathrm{crit}}=\mu_{\mathrm{eff}}\left|F_{i j}^{\mathrm{n}}\right|
$$

where $\mu_{\text {eff }}$ is the effective friction coefficient. In the subcritical case, where $\left|F_{i j}^{\mathrm{s}}\right|<F_{i j}^{\mathrm{s}, \text { crit }}$, the sliding force is given by: ${ }^{1}$ 


$$
F_{i j}^{\mathrm{s}}=-k_{\mathrm{T}}\left(\int_{t_{0}}^{t} \mathbf{v}_{i j}^{\mathrm{s}}(\xi) d \xi\right) \cdot \mathbf{t}_{i j}^{\mathrm{s}}
$$

where $k_{\mathrm{T}}$ is the tangential stiffness coefficient. The time integral on the right-hand side of Equation 5 describes the tangential elastic displacement from the beginning of the contact at time $t_{0}$ to current time $t$. When the connection between the particles $i$ and $j$ is broken, this integral becomes zero. According to Mindlin (1949), $k_{\mathrm{T}}$ can be computed from the following expression: ${ }^{4}$

$$
k_{\mathrm{T}}=8 G^{\mathrm{s}} a(t)
$$

where $G^{\mathrm{s}}=E / 2\left(1+\nu_{i}\right)$ is the shear modulus of the particles. The corresponding torque $\left(\mathbf{M}_{i}^{\mathrm{s}}\right)$ on the particle $i$ caused by the sliding resistance is given by:

$$
\mathbf{M}_{i}^{\mathrm{s}}=R_{\mathrm{p}}^{i} F_{i j}^{\mathrm{s}}\left(\mathbf{n} \times \mathbf{t}_{i j}^{\mathrm{s}}\right) .
$$

Another type of movement, which has to be taken into account when dealing with rotating particles, is the particle rolling. According to Marshall (2009), rolling is the dominant type of the tangential interaction for colloidal particles, due to their small inertia. For the derivation of rolling resistance we also adopt the spring-slider model as in the case of sliding. We first define the so-called rolling velocity $\left(\mathbf{v}_{i j}^{\mathrm{L}}\right)$, which is for particles $i$ and $j$ given by: ${ }^{5}$

$$
\mathbf{v}_{i j}^{\mathrm{L}}=R\left(\boldsymbol{\Omega}_{i}-\boldsymbol{\Omega}_{j}\right) \times \mathbf{n}
$$

The direction of rolling $\left(\mathbf{t}_{i j}^{\mathrm{L}}\right)$ is obtained from:

$$
\mathbf{t}_{i j}^{\mathrm{L}}=\frac{\mathbf{v}_{i j}^{\mathrm{L}}}{\left|\mathbf{v}_{i j}^{\mathrm{L}}\right|} .
$$

Finally the rolling resistance torque $\left(\mathbf{M}_{i}^{\mathrm{L}}\right)$ is postulated $\mathrm{as}^{5}$ : 


$$
\mathbf{M}_{i}^{\mathrm{L}}=-k_{\mathrm{L}} \tau^{\mathrm{L}}
$$

where $k_{\mathrm{L}}$ is the rolling coefficient and $\tau^{\mathrm{L}}$ is the rolling displacement obtained from:

$$
\tau^{\mathrm{L}}=\left(\int_{t_{0}}^{t} \mathbf{v}_{i j}^{\mathrm{L}}(\xi) d \xi\right) \cdot \mathbf{t}_{i j}^{\mathrm{L}},
$$

where $t_{0}$ and $t$ have the same meaning as in Equation 5. We refer to the cited papers for further explanation and derivation of the used formulas. Finally, the model of the rolling resistance is, similarly to the sliding resistance model, created by the connection of spring and slider. The critical rolling displacement $\left(\tau^{\mathrm{L}, \mathrm{crit}}\right)$, which limits the spring, can be computed from:

$$
\tau^{\mathrm{L}, \mathrm{crit}}=R \theta^{\mathrm{crit}}
$$

where $\theta^{\text {crit }}$ the critical rolling angle, which is the parameter of the model. If the value of the rolling displacement $\left(\tau^{\mathrm{L}}\right)$ exceeds $\tau^{\mathrm{L} \text {,crit }}$, then the critical value is used instead.

Twisting is the last type of tangential interaction used in the model. It is relevant only for the 3D model, since it occurs when two colliding particles have different rotation rates in the direction of $\mathbf{n}$. We can define the relative twisting rate $\left(\Omega_{\mathrm{T}}\right)$ as:

$$
\Omega_{\mathrm{T}}=\left(\Omega_{i}-\Omega_{j}\right) \cdot \mathbf{n} .
$$

The twisting resistance is, similarly to sliding and rolling, expressed in the form of spring and slider model. The twisting resistance torque is then: ${ }^{1}$

$$
M_{\mathrm{T}}=-k_{\mathrm{Q}} \int_{t_{0}}^{t} \Omega_{\mathrm{T}}(\tau) d \tau,
$$

where the torsional stiffness can be expressed as $k_{\mathrm{Q}}=k_{\mathrm{T}} a^{2} / 2$. The critical twisting torque is given as follows: 


$$
M_{\mathrm{T}, \mathrm{crit}}=\frac{2}{3} a F^{\mathrm{s}, \mathrm{crit}},
$$

and for the case when $\left|M_{\mathrm{T}}\right|>M_{\mathrm{T}, \text { crit }}$, the twisting resistance torque is given by:

$$
M_{\mathrm{T}}=-M_{\mathrm{T}, \text { crit }} \frac{\Omega_{\mathrm{T}}}{\left|\Omega_{\mathrm{T}}\right|} .
$$

The tangential forces and torques are added to the resulting force and torque vectors acting on each particle. The resulting force $\left(\mathbf{F}_{i}\right)$ and torque $\left(\mathbf{M}_{i}\right)$ acting on particle $i$ are obtained as follows:

$$
\begin{gathered}
\mathbf{F}_{i}=F_{\mathrm{N}} \mathbf{n}+F^{\mathrm{s}} \mathbf{t}^{\mathrm{s}}, \\
\mathbf{M}_{i}=R_{\mathrm{p}} F^{\mathrm{s}}\left(\mathbf{n} \times \mathbf{t}^{\mathrm{s}}\right)+M^{\mathrm{L}}\left(\mathbf{t}^{\mathrm{L}} \times \mathbf{n}\right)+M_{\mathrm{t}} \mathbf{n},
\end{gathered}
$$

where $F_{\mathrm{N}}$ is the resulting normal force.

\section{Hydrodynamics}

The body forces originating from the particles need to be accounted for in the computation of the fluid flow. For this purpose, the volume partitioning method was used and the schematics of this method is shown in Figure S1.

\section{Cutoff distance}

In methods employing particle dynamics, a cutoff distance for the interaction forces and torques is typically used to reduce the computational time. This cutoff distance $h_{\text {cut }}$ was used also in this work for lubrication forces both between the particles and between the particle and the wall. The default value of $h_{\text {cut }}=R_{\mathrm{p}}$ was chosen as the distance at which 


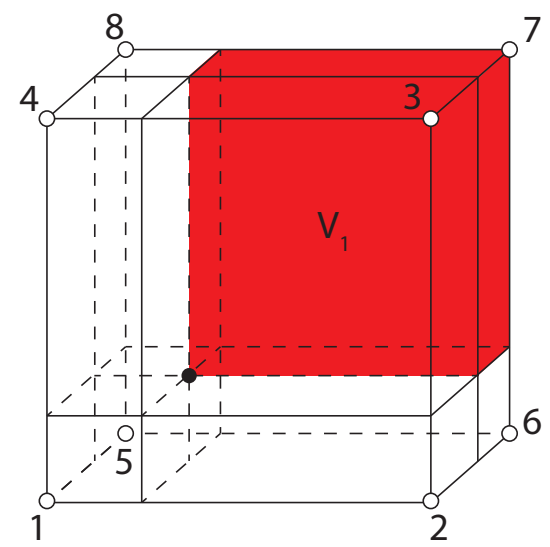

Figure S1: Schematics of the volume partitioning method used for the distribution of particle forces onto the fluid grid. The volume of the sub-grid cell $V_{1}$ opposite to the node 1 is highlighted by the red color. Adapted from Marshall (2009). ${ }^{1}$

the tangential and rotational contributions to lubrication reach zero (see Equations 12-19 in the main text). However, variation of the cutoff distance has an effect on the amount of dissipation in the system and thus on the suspension viscosity. This effect is demonstrated in Figure S2 for three different values of the cutoff distance. The Figure also includes $4 \%$ errorbars and it can be seen that the difference between the results for $h_{\text {cut }}=R_{\mathrm{p}}$ and $h_{\text {cut }}=3 R_{\mathrm{p}} / 2$ is almost within the error caused by fluctuations naturally occurring in the simulations.

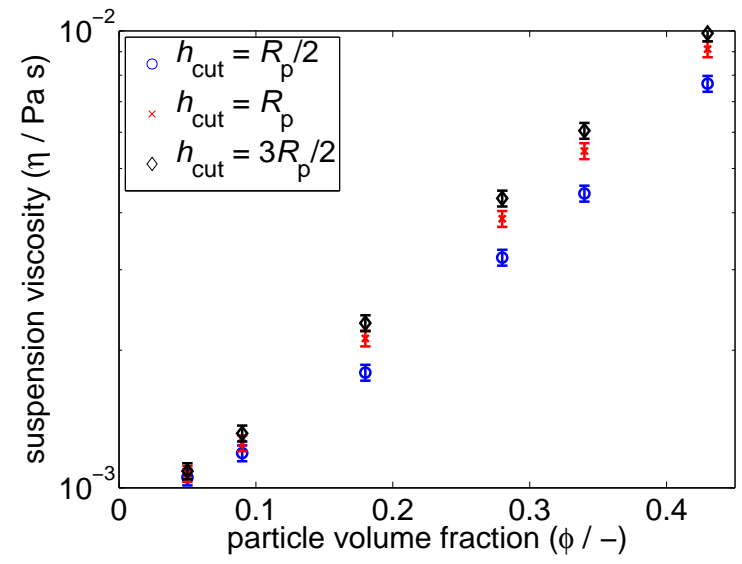

Figure S2: Steady-state viscosity as a function of particle volume fraction for different values of cutoff distance $h_{\text {cut }}$. 


\section{Chain of Particles}

The first comparison with literature results was performed for a simple situation of $N$ particles forming a one dimensional chain. If a force $F_{\mathrm{Ch}}$ is applied to one particle at the end of the chain in the direction towards the other particles, the whole chain should behave as a slender body and its velocity $U_{\mathrm{t}}$ can be obtained as a result of the simulation. ${ }^{6}$ For this simple situation, the velocity of the spheroidal rod $U_{\mathrm{SB}}$ can be obtained from slender-body theory $^{7}$ as follows:

$$
U_{\mathrm{SB}}=\frac{F_{\mathrm{Ch}}}{6 \pi \eta_{\mathrm{F}} R_{\mathrm{p}}} \frac{3}{2} \frac{\ln (2 N)-1 / 2}{N}\left[1+O\left(\frac{1}{N}\right)^{2}\right]
$$

When neglecting the term $O\left(\frac{1}{N}\right)^{2}$ in Equation 19, this equation provides a value of $U_{\mathrm{SB}}$ for $N \gg 1$. From the results shown in Figure S3, we can conclude that (except for $N=5$ ) our model provides predictions of $U_{\mathrm{t}}$ that lie within $10 \%$ error when compared with Equation 19, which is similar to the results obtained with Stokesian dynamics. ${ }^{6}$ The value of critical distance $\epsilon_{\mathrm{c}}$ used to calculate values of $U_{\mathrm{t}}$ in Figure S3 was taken from the analysis that is presented in the following sections.

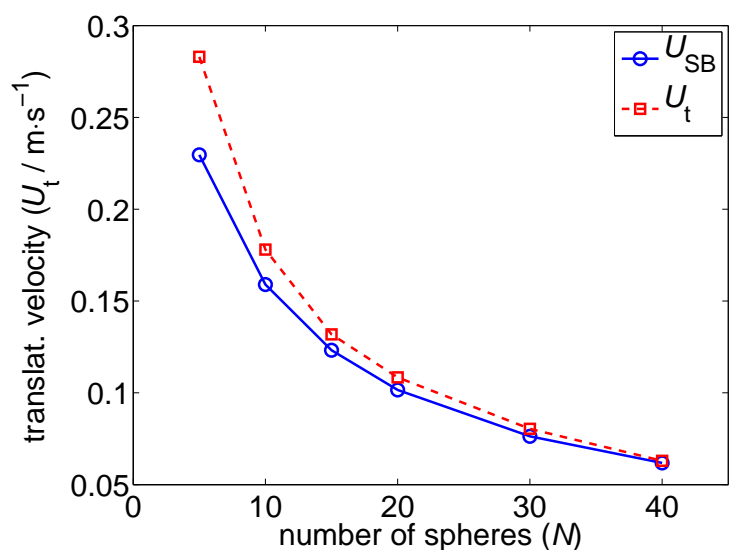

Figure S3: Translational velocity $U_{\mathrm{t}}$ as a function of the number of spheres in the chain compared with the prediction of Equation $19\left(U_{\mathrm{SB}}\right)$. The values of parameters were: $F_{\mathrm{Ch}}=$ $1 \mathrm{nN}, \epsilon_{\mathrm{c}}=0.1$ and $R_{\mathrm{p}}=125 \mathrm{~nm}$. 


\section{Constraining Lubrication Forces}

The correction that is used to constrain lubrication forces affects the shape of the forcedistance curves. This is demonstrated in Figure S4, where the normalized force is plotted against the separation distance between two particles. It is clear that the increasing critical distance $\epsilon_{\mathrm{c}}$ leads to the decreasing magnitude of the lubrication forces.

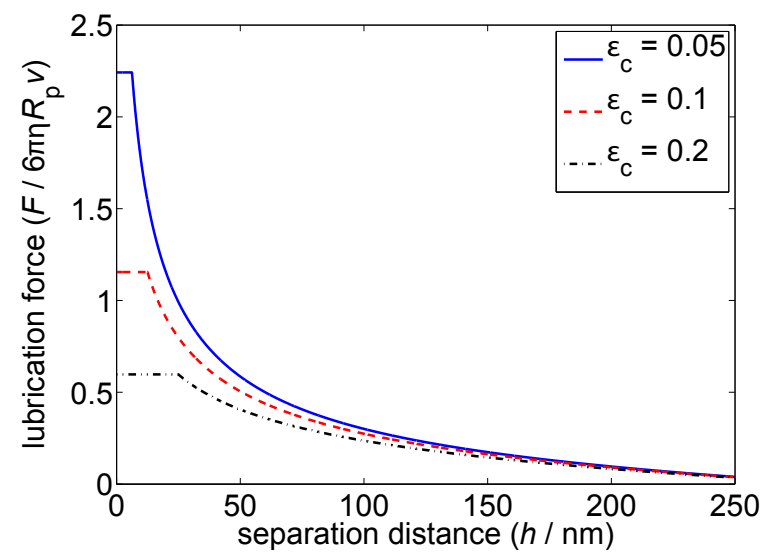

Figure S4: Normalized lubrication force as a function of separation distance $h$ for different values of critical distance $\epsilon_{\mathrm{c}}$. The particle radius was $R_{\mathrm{p}}=125 \mathrm{~nm}$.

In addition to the comparison with experimental data, which is shown in the main text, we present Figure S5 with the results of our simulations for different values of the critical distance $\epsilon_{\mathrm{c}}$ compared with the predictions of Equations of Krieger \& Dougherty, Maron \& Pierce and Mendoza \& Santamaria-Holek (Equations 1, 2 and 3 in the main text). The value of the maximum packing fraction $\phi_{\mathrm{m}}=0.70$ was chosen as a compromise between values used in literature for the high-shear limit for viscosity. Commonly used values for $\phi_{\mathrm{m}}$ range between 0.68 and 0.74 (fcc close-packing of monodisperse spheres). ${ }^{8-10}$ The results in Figure S5 clearly support the choice of $\epsilon_{\mathrm{c}}=0.1$, which was made in the main text. 


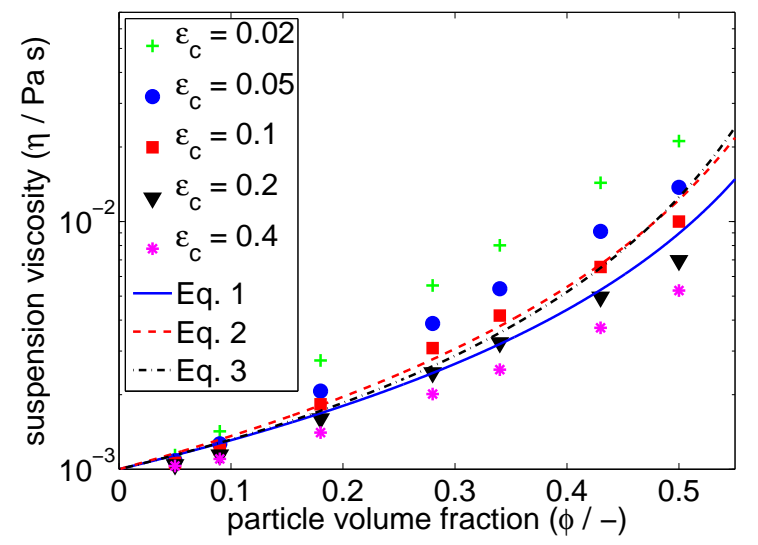

Figure S5: Steady-state viscosity as a function of $\phi$ for different values of critical distance $\epsilon_{\mathrm{c}}$ compared with the theoretical prediction by the equations of Krieger \& Dougherty, Maron \& Pierce and Mendoza \& Santamaria-Holek (Equations 1, 2 and 3 in the main text) with the maximum packing fraction $\phi_{\mathrm{m}}=0.70$. The values of parameters were: $G=400000 \mathrm{~s}^{-1}$ and $R_{\mathrm{p}}=125 \mathrm{~nm}$. 


\section{References}

(1) Marshall, J. S. Discrete-element modeling of particulate aerosol flows. J. Comput. Phys. 2009, 228, 1541-1561.

(2) Becker, V.; Briesen, H. Tangential-force model for interactions between bonded colloidal particles. Phys. Rev. E: Stat., Nonlinear, Soft Matter Phys. 2008, 78, 061404.

(3) Harshe, Y. M.; Lattuada, M. Breakage Rate of Colloidal Aggregates in Shear Flow through Stokesian Dynamics. Langmuir 2012, 28, 283-292.

(4) Mindlin, R. D. Compliance of Elastic Bodies In Contact. J. Appl. Mech. 1949, 16, 259-268.

(5) Marshall, J. S. Particle aggregation and capture by walls in a particulate aerosol channel flow. J. Aerosol Sci. 2007, 38, 333-351.

(6) Brady, J. F.; Bossis, G. Stokesian Dynamics. Annu. Rev. Fluid Mech. 1988, 20, 111157.

(7) Chwang, A. T.; Wu, T. Y. T. Hydromechanics of Low-reynolds-number Flow .2. Singularity Method For Stokes Flows. J. Fluid Mech. 1975, 67, 787-815.

(8) Krieger, I. M. Rheology of monodisperse latices. Advances in Colloid and Interface Science 1972, 3, 111-136.

(9) Mendoza, C. I.; Santamaria-Holek, I. The rheology of hard sphere suspensions at arbitrary volume fractions: An improved differential viscosity model. Journal of Chemical Physics 2009, 130, 044904.

(10) de Kruif, C. G.; van Iersel, E. M. F.; Vrij, A.; Russel, W. B. Hard-sphere Colloidal Dispersions - Viscosity As A Function of Shear Rate and Volume Fraction. Journal of Chemical Physics 1985, 83, 4717-4725. 